\title{
Matteo Bianchi
}

Università di Bergamo

\section{Gli alberi della savana: le sopravvivenze del passato}

doi: 10.7358/ling-2015-001-bian

matteobianchi02@libero.it

La tesi e l'antitesi convivono con la sintesi: ecco la vera trinità dell'uomo né prelogico, né logico, ma reale.

(Pier Paolo Pasolini, Maria Callas)

Il film-documentario Appunti per un'Orestiade africana rappresenta, dal mio punto di vista, un nodo fondamentale nella poetica pasoliniana. Infatti negli Appunti Pasolini riprende il mito antico, che aveva già approfondito in Edipo Re sostituendo così il mito materno e originario de La meglio gioventù e quello moderno del sottoproletariato romano in Ragazzi di vita e una Vita violenta, e anticipa alcune riflessioni sulla società contemporanea che verranno sviluppate ampiamente in Scritti corsari, Lettere luterane e in Petrolio. Si può affermare che gli Appunti siano l'ultimo progetto di "utopia della sintesi", tanto cara a Pasolini, che negli anni Settanta verrà sostituita da un'idea pessimistico-apocalittica sulla società e gli esseri umani.

Appunti per un'Orestiade africana avrebbe dovuto far parte di un film sul Terzo mondo diviso in cinque episodi: l'India, l'Africa nera, i Paesi arabi, l'America del Sud e i Ghetti neri degli Stati Uniti. Fin dall'inizio degli anni sessanta Pasolini delinea l'Africa come un modello alternativo allo sviluppo occidentale:

Adopero questa parola [Bandung] in tutta l'estensione del suo significato, ivi compresa anche la rinascita, la lotta per la rinascita, la strada da percorrere per raggiungerci quaggiù nella nostra magnifica storicità. Adopero questa parola implicandovi anche la guerra dei sottoproletariati algerini, angolani, kikuyu ... Gandhi, Kenyatta ... Adopero questa parola implicandovi anche il Ghana, che fra pochi decenni sarà ricco come la Svizzera ... Ma adopero soprattutto questa parola come senhal geografico per comprendervi la fisicità dei "regni della fame", il "fetore di pecora del mondo che mangia i suoi prodotti". ( $\mathrm{Pa}$ solini $1999,1572-73$ ) 
L'idea di un Terzo Mondo che comprende anche l'Italia configura il terzomondismo pasoliniano come la ricerca di un'autentica alterità culturale (Carminati 2007, 4). Pasolini così si fa portavoce di universalismo transnazionale (Casarino 2010, 673-96 e Jaran 2013, 49-64):

L'Italia è [...] un paese da laboratorio, perché in essa coesistono il mondo moderno industriale e il Terzo Mondo. Non c'è differenza fra un villaggio calabrese e un villaggio indiano o marocchino, si tratta di due varianti di un fatto che al fondo è lo stesso. $\mathrm{E}$ in Italia abbiamo visto che il contadino calabrese il suo mondo lo perde, non lo conserva e non ne ricava qualcosa da suggerire. (Pasolini 1999, 1638)

Con questo bagaglio ideologico Pasolini parte alla volta dell'Africa per girare i suoi appunti.

Inizialmente aveva in mente di riprodurre la sceneggiatura de Il padre selvaggio, ma il carattere letterario di questo testo e, di conseguenza, la difficoltà nel riprodurlo cinematograficamente gli fanno optare per l'Orestiade di Eschilo. Pasolini conosceva bene la tragedia eschilea: nel 1960 l'aveva tradotta su richiesta di Vittorio Gassmann. Inoltre si deve dire che l'incontro con l'Orestiade rimane per Pasolini un punto fermo nell'evoluzione del proprio pensiero (Casi 2006, 78). Aveva reinterpretato liberamente la tragedia antica, che aveva assunto un valore profondamente antiletterale. Così anche il film riprende la rielaborazione ideologico-politica della trilogia eschilea:

Il significato delle tragedie di Oreste è solo, esclusivamente, politico. Clitemnestra, Agamennone, Egisto, Apollo, Atena, oltre che essere figure umanamente piene, contraddittorie, ricche, potentemente indefinite sono soprattutto dei simboli: o degli strumenti per esprimere scenicamente delle idee, dei concetti: insomma, in una parola, per esprimere quella che oggi chiamiamo una ideologia. (Pasolini 1988, 177)

In particolare l'Africa, reduce dall'esperienza coloniale, si trova agli occhi di Pasolini in una situazione di transizione tra un mondo arcaico originario e uno sviluppo democratico fondato sull'adozione di modelli economici, politici e culturali estranei alla cultura africana: quello liberista anglosassone e quello marxista filocinese. Pasolini vuole raccontare in chiave ideologica questo fondamentale passaggio.

L'immagine iniziale è di primaria importanza per comprendere il film: $\mathrm{Pa}$ solini riprende il suo viso che si specchia in una vetrina di un negozio (Fig. 1). In questa sorta di sdoppiamento si racchiude tutta l'idea che vuole sviluppare negli Appunti. Quando l'immagine si allarga e scompare il viso del regista, ci si rende conto che da una parte Pasolini guarda affascinato dentro la vetrina del negozio che vende frigoriferi, simbolo del capitalismo occidentalizzato e dell'americanizzazione della società. Dall'altra invece il suo sguardo si sofferma su alcuni alberi che si muovono seguendo il vento africano (Fig. 2). 


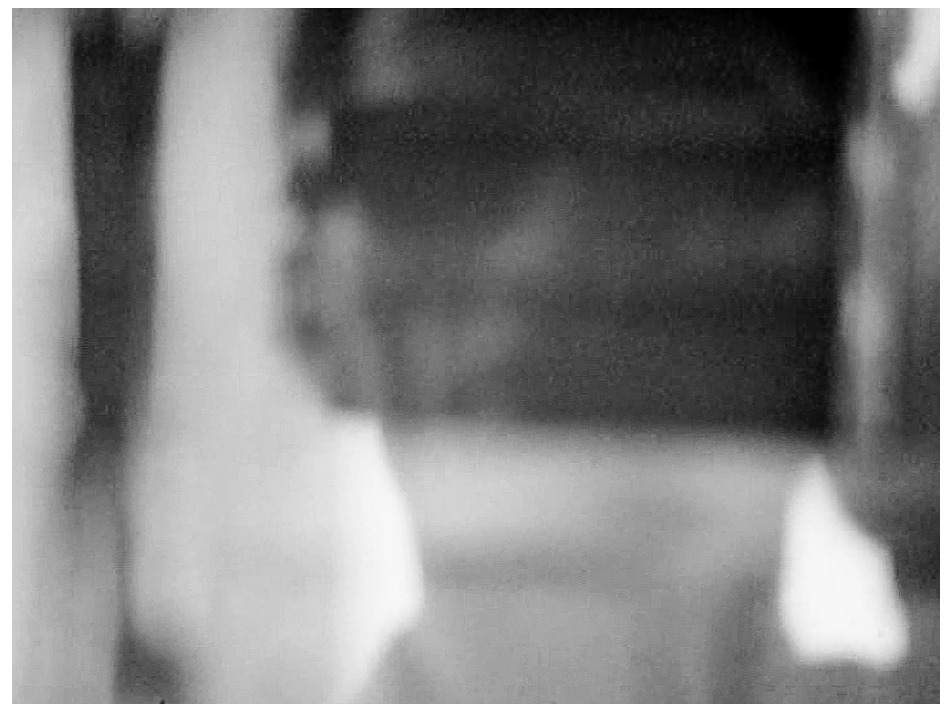

Fig. 1 - Appunti per un'Orestiade africana, 1970. 00:53.

(Per gentile concessione della Fondazione Cineteca di Bologna)

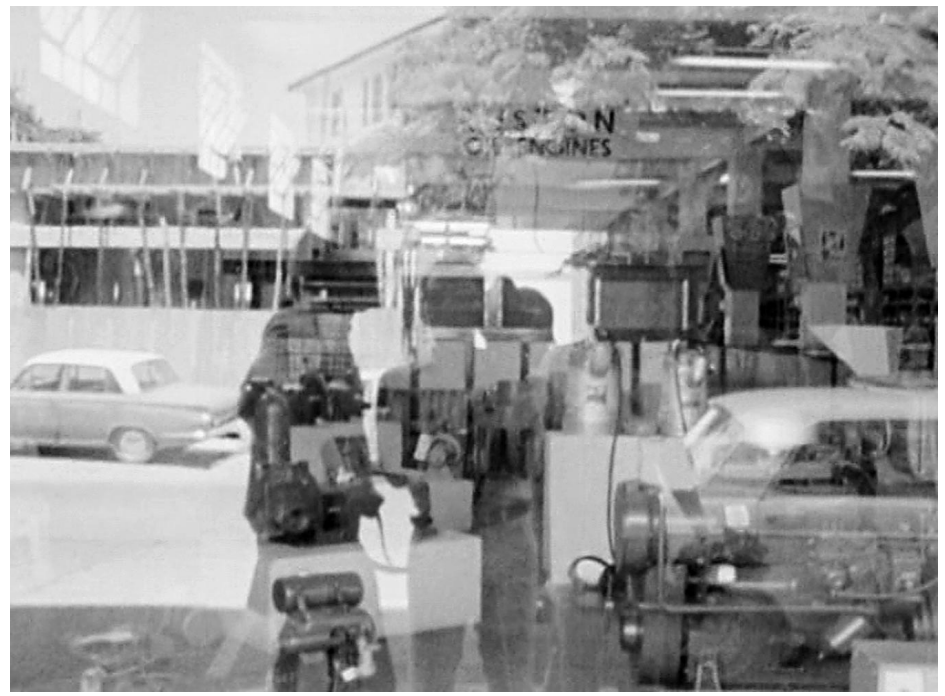

Fig. 2 - Appunti per un'Orestiade africana, 1970. 01:02.

(Per gentile concessione della Fondazione Cineteca di Bologna) 
Con questa immagine Pasolini mette in scena la profonda contraddizione dell'uomo contemporaneo in lotta tra mondo passato e mondo futuro. Inoltre questo sdoppiamento può essere interpretato come una dichiarazione di intenti del poeta, l'immagine dà consistenza all'idea che Pasolini ha di contemporaneità. Con la proiezione del proprio io, che si distacca per vedere e comprendere meglio la situazione africana, Pasolini ci mostra un punto di vista obliquo, subalterno, marginale e inattuale del contemporaneo. Da questo punto di vista Pasolini riprende la Seconda Considerazione Inattuale di Nietzsche, il quale situava la sua contemporaneità rispetto al presente in una sconnessione e in una sfasatura. Non è un caso che sia proprio Giorgio Agamben a recuperare questo tipo di contemporaneità. In un suo pamphlet dal titolo Che cos'è il contemporaneo? il filosofo scrive:

Appartiene veramente al suo tempo, è veramente contemporaneo colui che non coincide perfettamente con esso né si adegua alle sue pretese ed è perciò, in questo senso, inattuale; ma, proprio per questo, proprio attraverso questo scarto e questo anacronismo, egli è capace più degli altri di percepire e afferrare il suo tempo. (Agamben 2008, 8-9)

È proprio lo sdoppiamento del poeta davanti alla vetrina del negozio africano che rende esplicita questa inattualità. La contemporaneità diventa così una singolare relazione col proprio tempo, che aderisce a esso e, insieme, ne prende le distanze. Questa idea di un contemporaneo inattuale viene giustapposta ad una seconda definizione di contemporaneità data da Agamben, che risulta utile per interpretare l'immagine di Pasolini che si specchia:

Contemporaneo è colui che tiene fisso lo sguardo nel suo tempo, per percepirne non le luci, ma il buio. Tutti i tempi sono, per chi ne esperisce la contemporaneità, oscuri. Contemporaneo è colui che sa vedere questa oscurità, che è in grado di scrivere intingendo la penna nella tenebra del presente. (Ibid., 13)

Parafrasando Franz Kafka, si può affermare che Pasolini è contemporaneo perché si assume potentemente il negativo del proprio tempo, in questo caso messo in luce dal contesto storico e sociale africano degli anni Sessanta. In questo modo il poeta percepisce il buio del suo tempo come qualcosa che lo riguarda in profondità e non cessa di interpellarlo, diventando un qualcosa che si rivolge direttamente e singolarmente a lui. Proprio questo rapporto strabico, obliquo e marginale col presente è una delle maggiori risorse degli Appun$t i$, infatti è proprio con uno sguardo subalterno che Pasolini si accorge degli alberi dietro di lui.

Così, fin da subito, è evidente che gli alberi saranno i veri protagonisti del film, così come mostra l'inattualità pasoliniana e alcune inquadrature iniziali: la camera da presa si sposta bruscamente sugli alberi mentre Pasolini spiega di vo- 
ler girare l'Orestiade nell'Africa attuale. Gli alberi si stagliano anche dietro ad un masai che potrebbe essere Agamennone e dietro il probabile Pilade (Figg. 3 e 4).

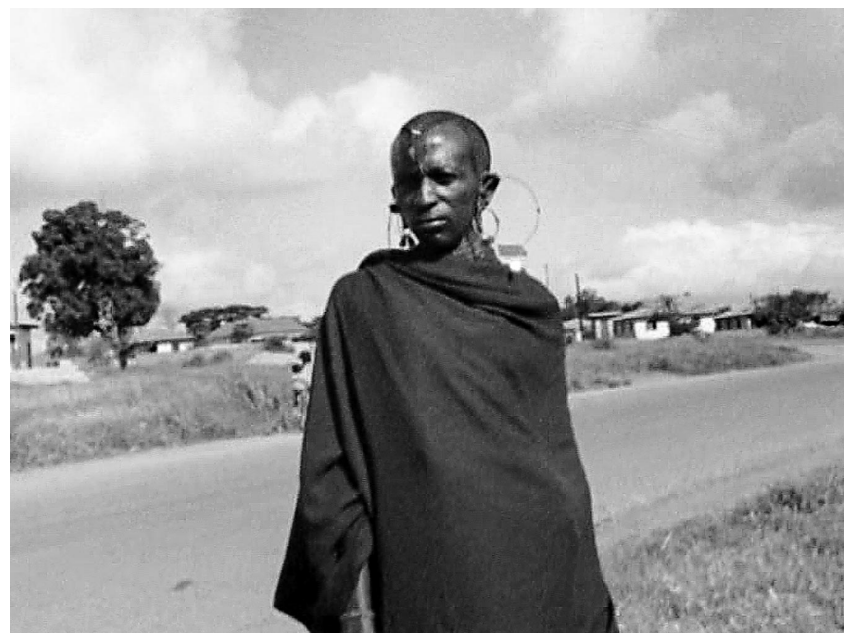

Fig. 3-Appunti per un' Orestiade africana, 1970. 05:26.

(Per gentile concessione della Fondazione Cineteca di Bologna)

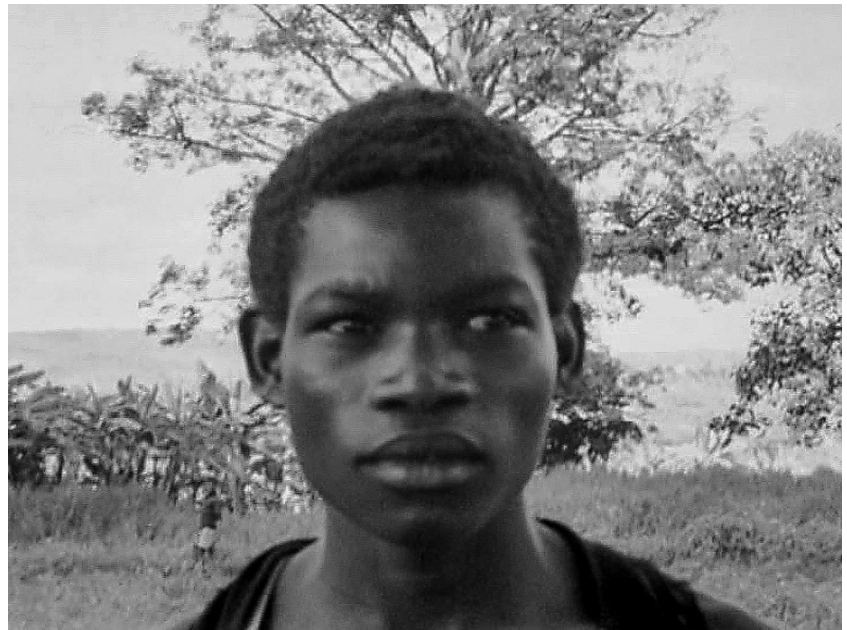

Fig. 4 - Appunti per un'Orestiade africana, 1970. 05:32.

(Per gentile concessione della Fondazione Cineteca di Bologna) 
Lidea di sdoppiamento, rafforzata dai primi piani che Pasolini realizza su diversi ragazzi africani, attraverso i quali vede un se stesso diverso, si coniuga con una riflessione sul tempo e, più in generale sulla storia.

In effetti Appunti per un'Orestiade africana può essere interpretato alla luce del paradigma filosofico-storiografico della traccia, così come è stato definito da Paul Ricoeur nel terzo volume del suo Tempo e racconto (Ricoeur 1991, 213-40). Negli ultimi anni si sono moltiplicati gli studi su questo film, di cui si è valutata l'enorme importanza all'interno dell'opera pasoliniana. Interpretandolo attraverso la teoria storiografica di Paul Ricoeur, vorrei inserirmi nella linea di studi tracciata da Manuele Gragnolati che ha letto gli Appunti attraverso le figure multistabili di Ludwig Wittgenstein. In quel caso le Kippbilder wittgensteiniane, che oscillano continuamente da un aspetto all'altro, servivano per spiegare il rapporto tra l'Io e l'Altro nei diversi aspetti in cui si mostrava negli Appunti, ad esempio Occidente contro Africa e progresso contro attaccamento irrazionale al passato (Gagnolati 2012, 119-33). Nel mio caso Ricoeur serve per una riflessione sul concetto di storia e di tempo nella poetica pasoliniana.

Per Ricoeur, la storia è una conoscenza per tracce, ciò significa fare appello al significato di un passato compiuto ma che resta preservato nelle sue vestigia. Il filosofo francese, per dare una soluzione al problema del valore mimetico della traccia e al sentimento di debito nei confronti del passato, analizza il concetto di passato mediante i tre generi platonici di Medesimo, Altro e Analogo, come vennero elaborati nel Sofista (Platone 2008, 179-211, 254b259 d). Ciascuno dei tre momenti è rappresentato dai tentativi più rilevanti della filosofia della storia e il passaggio, da una di queste posizioni filosofiche ad un'altra, risulta dall'impossibilità di ciascuna a risolvere in modo unilaterale ed esaustivo l'enigma della rappresentazione storica. Nella sua visione poetica della storia, mi sembra che Pasolini riconsideri il passato sotto il segno del Medesimo e sotto quello dell'Altro: gli alberi, come vedremo, rappresentano entrambe queste definizioni di passato.

Il passato può essere interpretato attraverso il genere del Medesimo perché è intelligibile solo se persiste nel presente, così la presenza degli alberi in molte delle inquadrature degli Appunti rendono visibile il fatto che l'antichità tribale dell'Africa persista nell'Africa decolonizzata. Pasolini ha una concezione della storia come rieffettuazione del passato, rendendo così possibile la trasposizione del mito greco antico nell'Africa contemporanea:

Nei miei film storici non ho mai avuto l'ambizione di rappresentare un tempo che non c'è più: se ho tentato di farlo, l'ho fatto attraverso l'analogia: cioè rappresentando un tempo moderno in qualche modo analogo a quello passato. Ci sono ancora dei luoghi del terzo mondo dove si fanno dei sacrifici umani: e ci sono ancora tragedie dell'inadattabilità di una persona del terzo mondo al mondo moderno: è questo persistere del passato nel presente che si può rappresentare oggettivamente. 
[...] Il passato diviene una metafora del presente: in un rapporto complesso, perché

il presente è l'integrazione figurale del passato. (Pasolini 1970, 172-73)

Da queste parole sembra che Pasolini avesse conoscenza del pensiero di Ernst Bloch, in particolare della sua idea di non-contemporaneità del contemporaneo, che in realtà aveva già teorizzato Erodoto nel V secolo a.C.:

L'esperienza dell'attualità non è la stessa per tutti. Alcuni vivono il presente solo esteriormente, per il semplice fatto che li si può vedere oggi. Ma ciò non vuol dire che essi vivano nello stesso tempo degli altri. Essi portano invece con sé qualcosa di anteriore che viene a mescolarsi con il presente. Il modo in cui un uomo vive il tempo dipende dal luogo in cui esso si trova in carne e ossa e soprattutto dalla classe alla quale appartiene. Epoche più antiche di quella attuale continuano a vivere nei ceti più antichi. (Bloch 1992, 82-83)

In Appunti per un'Orestiade africana Pasolini indossa le vesti dell'antropologo e ricerca le tracce del passato nel presente, delle sopravvivenze del passato nel mondo contemporaneo. Grazie a questa visione del passato, egli annulla la distanza temporale tra passato e presente, tentando di rappresentare la seguente idea: il processo di decolonizzazione dell'Africa non deve portare alla distruzione totale del proprio passato sacro e tribale, ma alla convivenza con la modernità in un rapporto di fratellanza dialettica. Il sentimento della storia di Pasolini è distante da posizioni storicistiche, accusate di procedere per addizione fornendo così una massa di fatti per riempire il tempo omogeneo e vuoto (Benjamin 1995, 85). Le uniche due forme di periodizzazione accettate sono quella della rivoluzione agricola, da cui deriva la civiltà arcaico-contadina, e quella industriale che, nel sottomettere la natura ai bisogni della produzione, segna un radicale cambiamento nel rapporto uomo-natura. La civiltà industriale però non assorbe la prima, identificata da Pasolini con la tradizione e il mito, ma vi si sovrappone come stratificazione superiore lasciandone intatti i dati originari (Calabrese Conti 1994, 44-45). In questo modo si evita una concezione progressiva della storia che invece è modellata, come una formazione geologica, da una variegata stratificazione di tempi. Così la tradizione, appartenendo alla sfera del sacro, rimane irriducibile a quella profana e si sottrae ad ogni possibile processo di secolarizzazione della storia (Löwith 2010) ${ }^{1}$. L'utopia pasoliniana non lascia spazio ad alcuna Aufhebung, quindi a nessun superamento, ma solo ad una sintesi di passato-presente che restano inalterati. $\mathrm{Pa}$ -

1 Karl Löwith mette in risalto il fatto che la nozione moderna di storia ha la sua origine nella prospettiva escatologicca giudaico-cristiana e giunge, tuttavia, alla secolarizzazione di tale progetto così lo spirito moderno appare derivato dal cristianesimo, ma anticristiano nel suo sviluppo.

Linguce \& - 1/2015

http://www.ledonline.it/linguae/ 
solini afferma il suo totale dissenso nei confronti del pensiero hegeliano: per lui prevale una visione ieratica e immobile della realtà. La sua concezione della storia è antidialettica: "La tesi e l'antitesi convivono con la sintesi: ecco la vera trinità dell'uomo Né prelogico, né logico, ma reale” (Pasolini 2003, 262).

Più volte Pasolini ha sottolineato l'inadeguatezza della dialettica hegeliana, come durante l'intervista con Jean Duflot:

Da cosa nasce la "speranza", quella della prassi marxista e quella della pragmatica borghese? Nasce da una comune matrice: Hegel. Io sono contro Hegel (esistenzialmente - empirismo eretico). Tesi? Antitesi? Sintesi? Mi sembra troppo comodo. La mia dialettica non [è] più ternaria ma binaria. Ci sono opposizioni, inconciliabili.

Hegel! Sade! Il mito! Eh già! Quando parlo della natura bisogna sempre intendere "mito della natura": mito antihegeliano e antidialettico, perché la natura non conosce i "superamenti". Ogni cosa in essa si giustappone e coesiste [...]. In quanto "storicista”, capisco che la storia è una evoluzione, un continuo superamento dei dati; sono altrettanto consapevole però che tali dati non vengono mai eliminati, ma sono permanenti. Sarà irrazionale, ma è così. (Pasolini 1999, 1461 e 1474)

Il documentario diventa così una chiave di interpretazione della realtà contemporanea. Secondo Pasolini, il testo di Eschilo è attualizzabile nell'Africa degli anni sessanta, perché come Oreste abbandona Argo con la sua cultura tribale e irrazionale giungendo ad Atene, dove scopre la ragione e la democrazia, così gli stati africani, decolonizzandosi e superando la loro cultura ancestrale, conquistano l'indipendenza e la democrazia avviandosi a diventare moderni.

Nel film un'immagine è emblematica per comprendere la funzione svolta dagli alberi nello spiegare la compresenza del mondo antico nel mondo moderno. Verso la fine del film alcuni alberi, simbolo della tradizione tribale africana, si stagliano davanti all'università di Dar Es Saalam che nell'idea di Pasolini avrebbe dovuto rappresentare Atene, simbolo della razionalità (Fig. 5).

Questo processo metonimico è una costante nel cinema pasoliniano, basti pensare ad un'immagine del film Accattone: Stella tra le bottiglie. Come ha sostenuto durante un'intervista, le bottiglie erano un chiaro riferimento a Giorgio Morandi, in particolare nell'interpretazione datane dal suo allievo Francesco Arcangeli. Nella poetica pasoliniana Stella ha la stessa funzione delle bottiglie vuote e delle nature morte nella poetica di Morandi. Nel pittore bolognese è proprio attraverso le bottiglie, nelle forme degli oggetti nel loro intrattenere la luce, che l'Italia della provincia ottocentesca entrava in contatto col mondo moderno. Così come Stella rappresenta un mondo perduto, quel "paese di temporali e primule", ossia il Friuli, tante volte descritto da Pasolini. Morandi, come del resto Pasolini, diventa il confine tra due epoche; lo stesso si può dire guardando l'immagine degli alberi che si stagliano davanti all'Università 


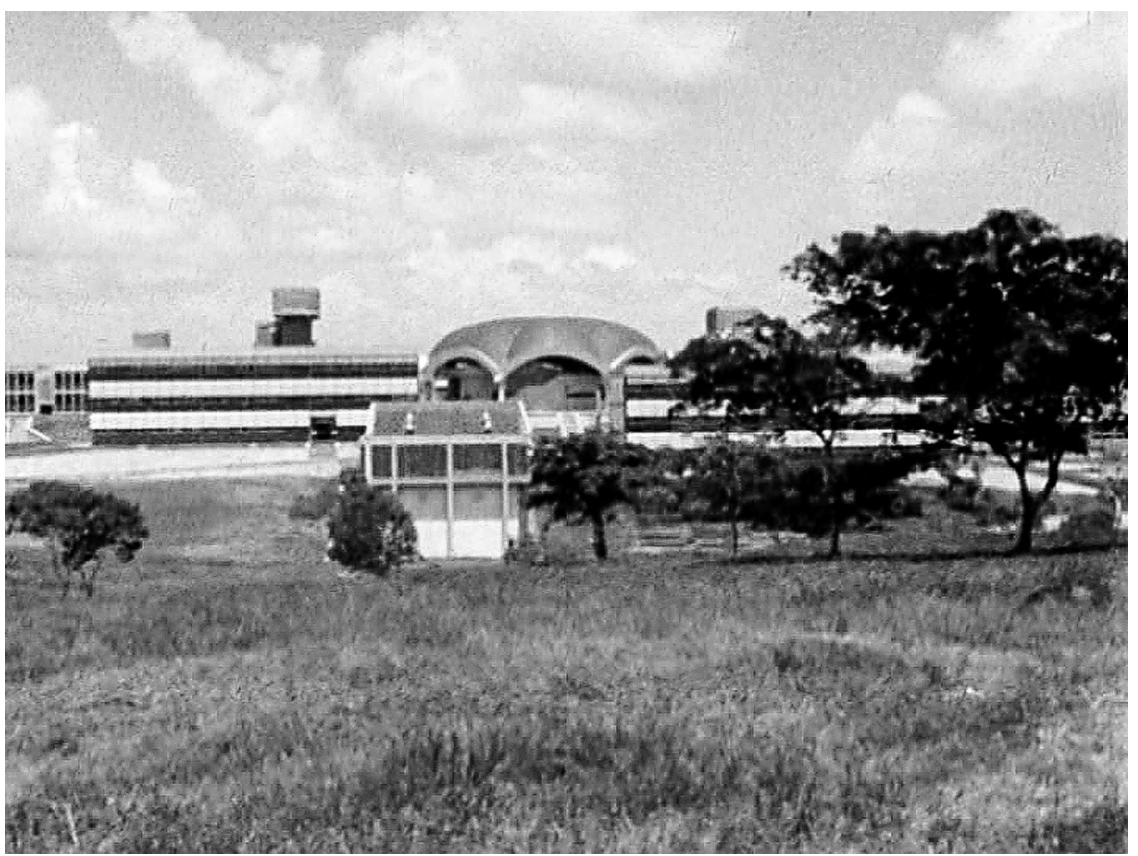

Fig. 5 - Appuntiper un'Orestiade africana, 1970. 47:47

(Per gentile concessione della Fondazione Cineteca di Bologna)

di Dar Es Saalam. Gli alberi appartengono ad un "tempo perduto", che ogni giorno di più cade in oblio, logorato, affranto, travolto dalla modernità. Da questo punto di vista è interessante l'accostamento nella Divina Mimesis tra la fotografia della chiesa di Casarsa e quella di un villaggio africano con in primo piano alcuni bambini, che rappresentano la giustapposizione di un elemento dell'infanzia friulana di Pasolini e della sua esperienza in Africa. Gli Appunti sono, così, l'ultimo tentativo di chiudere in circolare unità il dissidio insanabile tra anni quaranta-cinquanta e anni sessanta, vissuto da Pasolini come dissidio tra speranza e smarrimento, e per questo risultano fondamentali nell'opera pasoliniana.

Queste sopravvivenze del passato nel presente sviluppano anche una interpretazione ontologica negativa del passato, rendendo possibile spiegare il passato alla luce della categoria dell'Altro. Negli Appunti Pasolini, per considerare il passato come Altro, carica di grande importanza la trasformazione delle Erinni, le Furie irrazionali della città di Argo rappresentate dagli alberi, in Eumenidi, le divinità religiose che si presentano in sogno nella città di Atene. Ciò che Pasolini sostiene è che non è possibile cancellare del tutto il sacro 
e l'irrazionale - le Erinni - come invece vorrebbe chi sostiene una razionalità estremistica e un progresso senza sviluppo. La tradizione, anche se sublimata - le Eumenidi -, rimarrà sempre come traccia di un tempo originario che è impossibile annullare:

[...] certi elementi del mondo antico, appena superato, non andranno del tutto repressi, ignorati: andranno, piuttosto, acquisiti, riassimilati, e naturalmente modificati. In altre parole: l'irrazionale, rappresentato dalle Erinni, non deve essere rimosso (ché poi sarebbe impossibile), ma semplicemente arginato e dominato dalla ragione, passione producente e fertile. Le Maledizioni si trasformano in Benedizioni. Lincertezza esistenziale della società primitiva permane come categoria dell'angoscia esistenziale o della fantasia nella società evoluta. (Calabrese Conti 1994, 177-78)

In questo modo Pasolini riconosce l'importanza dell'alterità storica e, nello stesso momento in cui cancella la distanza tra passato e presente, restituisce tale distanza temporale. Questa strategia del distanziamento è messa in opera al servizio del tentativo di rigettare l'etnocentrismo occidentale tipico della storia tradizionale, diventa un metodo contrappuntistico per portare alla luce narrazioni alternative o nuove (Said 1998). Come nella storiografia postcoloniale, Pasolini critica l'immagine lineare e progressiva del tempo storico; così le tracce di questo passato ancestrale e tribale sono "narrate" dagli alberi della savana che diventano lo scarto, il residuo rispetto ai modelli occidentali di dominio operanti nel razionalismo illuministico e rappresentano la diacronicità temporale del mito:

Restano altri personaggi da ricercare: le Furie. Ma le Furie sono irrappresentabili sotto l'aspetto umano e quindi deciderei di rappresentarle sotto un aspetto non umano. Questi alberi, per esempio, perduti nel silenzio della foresta, mostruosi, in qualche modo, e terribili. La terribilità dell'Africa è la sua solitudine, le forme mostruose che vi si può assumere la natura, i silenzi profondi e paurosi. L'irrazionalità è animale. Le Furie sono le dee del momento animale dell'uomo. (Pasolini 2001, 1183)

Per una filosofia della storia fedele all'idea di differenza-scarto, gli alberi rappresentano il passato (Fig. 6), ossia ciò che manca, l'assenza pertinente come direbbe Paul Ricoeur. La cultura tradizionale deve essere valorizzata perché è espressione di un mondo antropologicamente autentico e non contaminato. Pasolini recupera un concetto in voga tra i populisti russi di fine Ottocento, letti dal poeta attraverso i due volumi curati da Franco Venturi e pubblicati da Einaudi nel 1952: il vantaggio dell'arretratezza (Walicki 1973). Il mondo africano, non corrotto dall'individualismo borghese, viene pensato come già dotato delle potenzialità per andare verso una modernità alternativa, più comunitaria rispetto a quella prevalente in Occidente. L'attenzione verso il passato, la tradizione e l'alterità deve essere fatta risalite alla lettura di Antonio Gramsci, che Pasolini scopre dopo aver partecipato alle lotte bracciantili in Friuli (San- 
tato 2012, 219-41), in particolare il Gramsci del Quaderno n. 25, scritto nel 1935, dal titolo Ai margini della storia e il Quaderno n. 27, sempre del 1935, Osservazioni sul "Folklore".

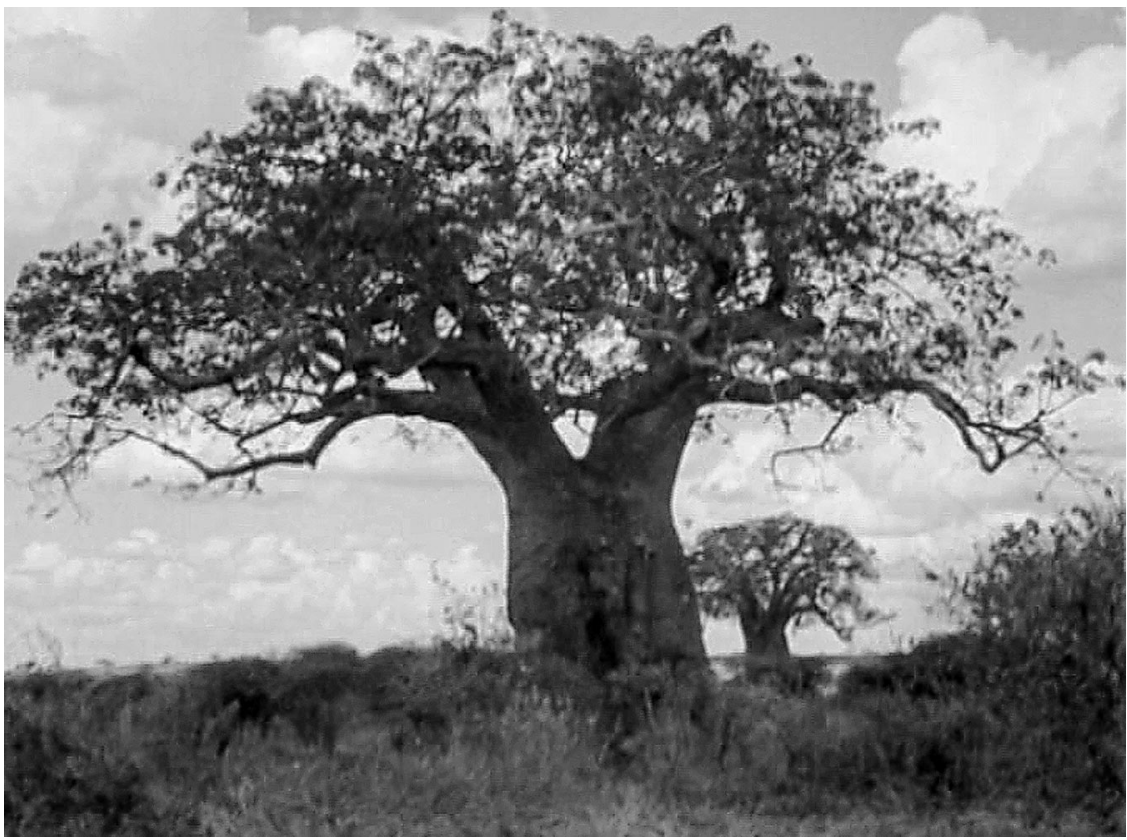

Fig. 6 - Appunti per un'Orestiade africana, 1970. 21:12.

(Per gentile concessione della Fondazione Cineteca di Bologna)

Nel primo Quaderno Gramsci si sofferma sul concetto di egemonia culturale, affermando come, per una élite sociale, gli elementi dei gruppi subalterni hanno sempre un qualcosa di barbarico e patologico. I gruppi subalterni subiscono sempre l'iniziativa dei gruppi dominanti, anche quando si ribellano e insorgono. Le ferite di questa battaglia si trovano sui loro corpi, sguardi e gesti, come mostrano alcune riprese di Pasolini negli Appunti che hanno una forte valenza antropologica, quella che George Didi-Huberman definirà un'antropologia delle sopravvivenze, ossia un memoriale dei corpi che resistono alla storia che li aliena:

In realtà anche quando paiono trionfanti, i gruppi subalterni sono solo in istato di difesa allarmata (questa verità si può dimostrare con la storia della Rivoluzione francese fino al 1830 almeno). Ogni traccia di iniziativa autonoma da parte dei gruppi 
subalterni dovrebbe perciò essere di valore inestimabile per lo storico integrale; da ciò risulta che una tale storia non può essere tratta che per monografie e che ogni monografia domanda un cumulo molto grande di materiali spesso difficili da raccogliere. (Gramsci 1975, 2283-84)

Gramsci crede che per ridare valore e forza alle classi subalterne, in questo caso agli africani resisi da poco indipendenti, concepite come un'alterità in seno alla società mondiale, si dovrebbe creare una vera e propria fusione tra intellettuali e popolo: gli intellettuali dovrebbero pretendere di rappresentare il popolo solo quando il rapporto è fondato su di un'adesione organica in cui il sentimento-passione diventa comprensione quindi sapere. Ancora negli Appunti Pasolini crede alle possibilità di un intellettuale organico, che si prenda su di sé le richieste e le difficoltà del popolo, ma sarà proprio a partire dagli anni Settanta che anche questa idea di intellettualità verrà meno nella sua poetica e nei suoi scritti.

Nel secondo Quaderno Gramsci afferma come occorra studiare il folclore, rappresentato negli Appunti dagli alberi, come concezione del mondo e della vita di determinati strati della società in contrapposizione con le concezioni del mondo ufficiali, o in senso più ampio delle parti colte della società storicamente determinate. Gramsci definisce il folclore come un agglomerato indigesto di frammenti di tutte le concezioni del mondo e della vita che si sono succedute nella storia, delle tracce dei quali proprio nel folclore si trovano i superstiti documenti mutili e contaminati (Gramsci 1975, 2311):

Conoscere il folclore significa pertanto per l'insegnante conoscere quali altre concezioni del mondo e della vita lavorano di fatto alla formazione intellettuale e morale delle generazioni più giovani per estirparle e sostituirle con concezioni ritenute superiori [..] È certo che per raggiungere il fine occorrerebbe mutare lo spirito delle ricerche folcloristiche oltre che approfondirle ed estenderle. Il folclore non deve essere concepito come una bizzarria, una stranezza o un elemento pittoresco, ma come una cosa che è molto seria e da prendere sul serio. Solo così l'insegnamento sarà più efficiente e determinerà realmente la nascita di una nuova cultura nelle grandi masse popolari, cioè sparirà il distacco tra cultura moderna e cultura popolare o folclore. Un'attività di questo genere, fatta in profondità, corrisponderebbe nel piano intellettuale a ciò che è stata la Riforma nei paesi protestanti. (Ibid., 2314)

Pasolini è unito all'opera di Gramsci dal concetto di oblio, ossia la paura per la scomparsa della cultura orale e del mondo subalterno, rappresentato degnamente dagli alberi della savana. Gli alberi rappresentano la flebile voce dell'A1tro, di questa cultura tribale africana, che corre il serio pericolo di essere trasformato in differenza dopo l'indipendenza, nel senso che colui che vive in un luogo altro e senza tempo viene inserito in una storia, rappresentata degnamente dall'università di Dar Es Saalam. Il pericolo è quello di non restituire 
all'Africa la propria storia, cosicché c'è il rischio reale che non venga riconosciuta come soggetto di storia, ma le venga assegnato un posto che è solo un punto in una storia non sua. Ciò che serve è una scrittura, in questo caso quella cinematografica che è l'unica lingua della Realtà secondo Pasolini, che riesca a riportare alla luce il senso dell'Altro. Gli Appunti pasoliniani si sono prefissi lo scopo di costruire un discorso dell'Altro che non si enunci come discorso sull'Altro, come ad esempio succederebbe se un indiano parlasse la lingua del sapere occidentale. Da questo punto di vista è molto interessante la scelta di far cantare il coro, invece che parlare, sulla musica degli schiavi africani in America, ossia la musica jazz. In questo modo il coro dà voce al silenzio traumatico di un'alterità. Pasolini, come il pensatore sardo, vede l'unica possibile salvezza in ciò che resiste alla modernità; il corpo popolare, rappresentato negli Appunti dagli innumerevoli primi piani, porta con sé una cultura subalterna, cosicché il folclore diventa una vera e propria cultura di contestazione che si oppone ai ceti dominanti (Lombardi 1980, 117-31), una contro-storia. Gli alberi rappresentano un tempo circolare e sfasato e devono essere intesi come alterità rispetto al tempo lineare e progressivo borghese.

Questo amore per il passato ha una motivazione ben precisa, che deve essere fatta risalire alla morte del fratello Guido, di cui Pasolini scrive all'amico Franco Farolfi:

Chiedi a me cosa sia la mia ombra, ma è facile risponderti; è l'assenza di mio fratello, che mi fa misurare non più intellettualmente ma concretamente la nostra vita insensata e mai risolta.

[...] La mancanza di mio fratello se non mi dà quella meraviglia insopportabile dei primi mesi, è ora entrata dentro di me, si è diffusa nel mio animo, deprimendo tutti gli slanci e i momenti della mia esistenza. Tutto si è colorato intorno a me di un colore squallido e pauroso. Vedo il fondo di ogni cosa. (Pasolini 1988, 203 e 228)

Per spiegare questa struggente lettera viene in soccorso lo studioso americano Dominick LaCapra, che analizza l'elaborazione del lutto mediante la distinzione di origine freudiana tra working through e acting out, riprendendola per la sua teoria storiografica. I due concetti riguardano due diverse modalità di rapportarsi al trauma: il working through costituisce il processo di interpretazione e rielaborazione degli eventi traumatici, nel senso di una risemantizzazione dell'accaduto, che apre nuove possibilità di giudizio critico intorno all'esperienza umana di chi ha subito il trauma. Mentre l'acting out, di cui è vittima lo stesso Pasolini, si configura come un processo di arresto, in cui il traumatizzato, ripetendo compulsivamente il passato, viene da esso bloccato e posseduto (LaCapra 1999, 696-727), come dimostrano poche righe di una sua lettera:

Proprio in questi giorni ero colpito da una violenta nostalgia per il nostro passato (il bel tempo che ritorna, gli alberi che mettono le foglie, i tavolini dei caffè all'aperto, 
i ragazzi che vanno in giro sulle biciclette che sembrano nuove), tutto mi ricorda le nostre antiche (ma non tanto) fini-scuole. Mi vengono in mente il Sasso, le grotte del Farneto, i calanchi. (Pasolini 1988, 41)

Gli alberi, vera e propria contro-storia dell'Africa e immagine di un tempo diverso, ossia quel passato che ricerca Pasolini dalla morte del fratello, sono già presenti ne $\mathrm{Il}$ padre selvaggio, dove sono, per il protagonista Davidson, il limes tra la cultura "bianca", ossia occidentale, razionalistica e tipica di un mondo borghese già industrializzato e la cultura "di colore", arcaica, popolare, preindustriale e preborghese:

Davidson cammina, solo, rapido, per la pista rossiccia, verso il suo villaggio. A un tratto - senza nessuna ragione - si accorge di qualcosa: vede, per la prima volta, la foresta del suo villaggio natale, che tante volte aveva visto, certo, nella sua infanzia.

È una scoperta che egli fa (guidato, forse, dalla coscienza che ne aveva preso col suo tema poetico a scuola ...) e si incanta a guardare, davanti a sé ... Non è che un'immagine qualsiasi dell'interno dell'Africa: col suo fondo rosa, su cui si disegnano i capricciosi e funerei profili degli alberi equatoriali. E i rumori, le voci bestiali. L'ansia preumana che vi regna, con la sua pace di morte. (Pasolini 1975, 31-32)

Gli alberi sono simbolo della ciclicità del tempo naturale, legato ai suoi valori e ai suoi riti. Non è un caso che Pasolini decida di riprendere un rito di preghiera per gli antenati, legato ad un concetto naturale e ciclico di morte che così non viene espulsa dal mondo percettivo dei viventi (Fig. 7). Come Gramsci, Pasolini è contrario alla totale distruzione di queste culture ed è convinto che il folclore non debba essere considerato alla stregua di una stranezza o di un elemento esotico, ma come qualcosa da prendere terribilmente sul serio.

Però fin dalle prime battute del film ci si rende conto di come risulti impossibile questa coabitazione tra le antiche divinità primordiali e il nuovo mondo della ragione e della libertà. Si pensi ad esempio alla reazione di alcuni degli studenti universitari africani dopo la visione del film. Oltre ad alcuni primi piani che catturano questi ragazzi mentre sorridono a denti stretti, chiaro intento da parte di Pasolini di mostrare come il suo progetto sia non solo datato ma ormai fuori luogo, è interessante ascoltare alcune delle risposte che questi studenti offrono al poeta. Molti non riescono a capire l'attinenza nel rappresentare il mito dell'Orestiade nell'Africa attuale, perché non vedono la connessione tra l'Africa tribale e quella moderna. Così l'immagine iniziale del viso di Pasolini, il suo sdoppiamento, assume un altro interessante significato, oltre a quelli mostrati all'inizio: Pasolini si mette davanti alla macchina da presa come a mostrare che è pronto a qualsiasi critica, già consapevole dell'inattualità, ma di conseguenza anche della drammatica contemporaneità, del suo progetto (Syrimis 2013, 557-69). 


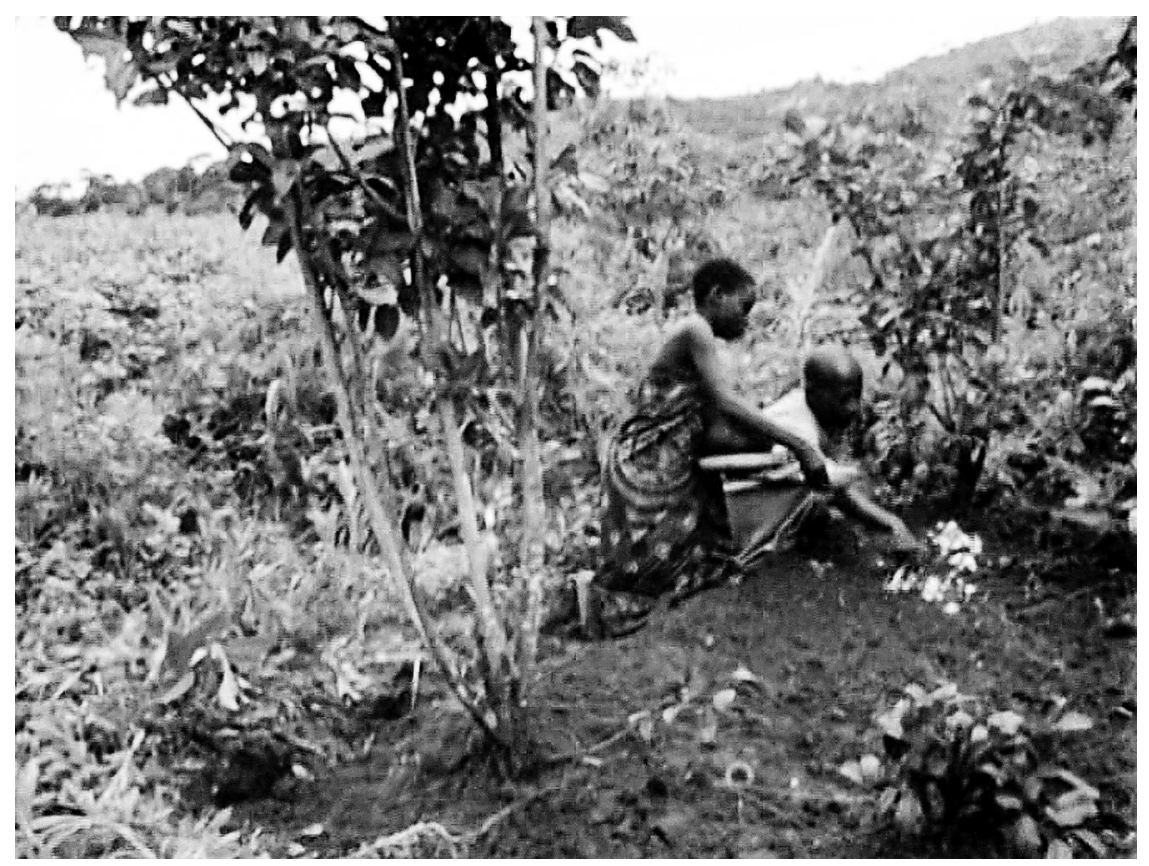

Fig. 7 - Appunti per un'Orestiade africana, 1970. 41:35.

(Per gentile concessione della Fondazione Cineteca di Bologna)

L'utopia pasoliniana della sintesi ${ }^{2}$ si dimostra irrealizzabile in un'altra riproduzione cinematografica del mito: Medea, realizzato un anno dopo gli Appunti. In questo caso Pasolini mostra come non ci sia alcuna soluzione al conflitto tra irrazionalismo, rappresentato da Medea, e razionalismo, di cui si fa portavoce Giasone. La scena finale è il punto di rottura di questo rapporto: Medea uccide i figli avuti da Giasone e brucia la casa. Queste fiamme, che cominciano a divampare dalla casa, rappresentano il fuoco ancestrale che dividerà per sempre il mondo primitivo di Medea da quello moderno di Giasone. La cinepresa riprende Giasone che corre verso il fuoco, mentre una spiritata Medea gli urla:

Perché cerchi di passare attraverso il fuoco? Non potrai farlo. È inutile tentare. Se vuoi parlarmi, puoi farlo, ma senza avermi vicino né toccarmi. (Pasolini 2001, 1288)

2 Anche in questo caso è percepibile la contraddizione insita nel discorso pasoliniano. Utilizza un termine derivante dalla filosofia hegeliana in termini antihegeliani. Infatti nella sua idea non è auspicabile alcun superamento della tesi e dell'antitesi, anzi la tesi e l'antitesi devono convivere. 
Giasone disperato chiede di poter piangere la morte dei figli innocenti, ma Medea chiosa in questo modo: "No, non insistere ancora. Ė inutile, niente è più possibile ormai" (ibid.).

Gli Appunti diventano così una vera e propria cesura nella linea di pensiero pasoliniana. Negli anni settanta prenderà piede una deriva apocalittica che culminerà con Salò o le 120 giornate di Sodoma, definitivo atto di accusa della società consumistica che ha sconfitto qualsiasi sopravvivenza del passato. Anche la Trilogia della vita, poi inevitabilmente abiurata, non rappresenta più uno sforzo propositivo di Pasolini nei confronti del passato, ma deve essere vista come un vero e proprio rifugio. Pasolini si nasconde in un passato ormai remoto, la cui vitalità e purezza non si trovano più nel mondo moderno che li ha completamente distrutti. L'utopia della sintesi non è più realizzabile e a Pasolini non resta che buttare il proprio corpo nell'arena: sarà da queste ceneri che nascerà Petrolio, vero e proprio testamento spirituale del poeta.

\section{BIBLIOGRAFIA}

Agamben, Giorgio. 2008. Che cos'è il contemporaneo? Roma: Nottetempo.

Benjamin, Walter. 1995. Angelus novus. Saggi e frammenti. Torino: Einaudi.

Bloch, Ernst. 1992. Eredità del nostro tempo. Milano: Il Saggiatore.

Calabrese Conti, Giuseppe. 1994. Pasolini e il sacro. Milano: Jaca Book.

Carminati, Luca. 2007. Orientalismo eretico: Pier Paolo Pasolini e il cinema del Terzo Mondo. Milano: Bruno Mondadori.

Casi, Stefano. 2006. Nel teatro della mia testa. Appunti, in Progetto Petrolio. Una giornata di studi sul romanzo incompiuto di Pier Paolo Pisolini. Bologna: Clueb.

Casarino, Cesare. 2010. "The Southern Answer: Pasolini, Universalism, Decolonization”. Critical Inquiry 36 (4): 673-96.

Gragnolati, Manuele. 2012. "Analogy and Difference. Multistable Figures in Pasolini's Appunti per un'Orestiade Africana", in The Scandal of Self-contradiction. Pasolini's Multistable Subjectives, 119-33. Wien-Berlin: Verlag Turia + Kant.

Gramsci, Antonio. 1975. Quaderni del carcere (vol. III). Torino: Einaudi.

Jaran, Mahmoud. 2013. "Pasolini, Fanon e l'umanesimo transnazionale". Studi Pasoliniani (7): 49-64.

LaCapra, Dominick. 1999. "Trauma, Absence, Loss”. Critical Inquiry (25): 696-727.

Lombardi, M. Luigi. 1980. Antropologia culturale e analisi della cultura subalterna. Milano: Rizzoli.

Löwith, Karl. 2010, Il significato della storia. I presupposti teologici della filosofia della storia. Milano: il Saggiatore. 
Murri, Serafino. 2003. Pier Paolo Pisolini. Milano: Il Castoro.

Pasolini, Pier Paolo. 1988. L'Orestiade. Torino: Einaudi. 2001. Per il cinema (vol. I). Milano: Arnaldo Mondadori. . 1975. Il padre selvaggio. Torino: Einaudi.

1970. "Il sentimento della storia”. Cinema Nuovo (205): 172-73. 1988. Lettere 1940-1954. Torino: Einaudi. 1999. Saggi sulla politica e sulla società. Milano: Arnaldo Mondadori. 2003. Tutte le poesie (vol. II). Milano: Meridiani Mondadori.

Platone, 2008. Sofista. Torino: Einaudi.

Ricoeur, Paul. 1991. Tempo e racconto (vol. III). Milano: Jaca Book

Said, Edward. 1998. Cultura e imperialismo. Roma: Gamberetti.

Syrimis, Michael. 2013. "Self-parody in Pasolini's La ricotta and Appunti per un'Orestiade africana", Forum Italicum 47 (3): 557-69.

Trento, Giovanna. 2010. Pasolini e l'Africa. Panmeridionalismo, e rappresentazioni dell'Africa postcoloniale. Milano: Mimesis.

Walicki, Andrzej. 1973. Marxisti e populisti: il dibattito sul capitalismo. Milano: Jaca Book.

\section{ABSTRACT}

In his work Pasolini shows great interest in the past and in otherness as represented by the farmers and labourers from Friuli, underclass Romans, and the poor of the Third World. This essay focuses on the documentary Appunti per un'Orestiade Africana, which can be interpreted as using the concept of the trace as it was theorized by the philosopher Paul Ricoeur in his Les tempes et récit, and illustrates what Pasolini meant by the idea of the survival of the past in the present. The aim of the essay is to demonstrate the importance of taking Appunti per un'Orestiade Africana as the turning point in $\mathrm{Pa}$ solini's career, marking the change from his early representations of the Friulian and the Roman to the later apocalyptic writings of Scritti corsari and Petrolio. 